\title{
Un cas de septicémie sporotrichosique
}

(avec démonstrations des cultures et des pièces microscopiques à la Société Impériale de Médecine de (Constantinople)

par

le Dr. Menahem Hodara,

chef du service dermatolegique de l'Hôpital do la Marine et

le Dr. Fuad Bey,

bactériologue de l'Hôpital de la Marine.

Messieurs! Permettez-nous, Messieurs, de vous communiquer la courte observation d'un cas de septicémie sporotrichosique, que nous avons étudié ensemble avec mon ami le bactériologue $\mathrm{Fuad} \mathrm{Bey}$, datant depuis un an et demi, avec fièvre continue, plus ou moins ondulante, durant depuis un an et des éruptions sporotrichosiques cutanées, datant depuis un an et quatre mois, généralisées dans tout le corps sous forme de papules et des nodules acneïformes, nécrotiques dont plusieurs sont vésiculo-pustuleuses, croûteuses ou par places exulcérées, laissant des petiles cicatrices et des pigmentations persistantes; at du côté des muqueuses des ulcérations amygdaliennes, des lésions érythémateuses, par places légèrement bulleuses aux muqueuses buccales et labiales et des lésions oculaires récidivantes sans cesse datant depuis un an. Le malade atteint de septicémie sporotrichosique est malheureusement, notre cher confrère, le distingué professeur I. D. B ey. Quoique souffrant, dans l'interêt de la science, il a consenti à faire photographier ses lésions, à permettre l'excision d'un nodule pour l'examen microscopique, et même il était décidé à venir ce soir à la Société pour que vous puissiez voir son cas excessivement Arch. f. Dermat. u. Syph. Bd. CX. 
curieux et rare mais le très maurais temps d'aujourd'hui l'a empêché. Nous lui exprimons ici notre très vive gratitude. Tout d'abord voici les notes qui nous ont été rémises textuellement par le professeur lui-même.

"La maladie m'a surpris au milieu de bonne santé habituelle, que j'ai presque toujours joui. Déjà depuis le commencement de juillet 1909 j'avais commencé à me sentir fatigué à la moindre fatigue. Bon marcheur d'habitude, je me sentais presque épuisé après avoir fait un petit trajet. En même temps je transpirais beaucoup. C'est le 26 juillet 1909 que j'ai eu pour la première fois un peu de fièvre $\left(37 \cdot 6^{\circ}\right)$. La nuit j'ai tellement transpiré, qu'on fut obligé de me changer deux fois le linge et même le lit. Le lendemain soir la fièvre fut un peu plus prononcée $38^{\circ}$ et le 3 jour j'avais $38^{\cdot} 5^{0}$. C'est alors que j'ai pris l'avis d'un confrère, qui me conseille de prendre un peu de quinine. Comme symptômes clinique je n'avais qu'un peu de courbature et une transpiration de plus en plus abondante. Pas de céphalée, pas de vomissement et pas de diarhée, ni constipation. Malgré la quinine que je prenais régulièrement la fièrre augmentait et chaque jour elle était plus prononcée que la veille; si bien que le $7 \mathrm{e}$ jour j'avais $39 \cdot 5^{\circ}$. Dès le 4e jour j'avais une douleur très vive du côté droit dans le dos. On a pris cela pour une pleurodynie; mais en réalité la douleur était localisée sur une côté. En pensant à une attaque de rhumatisme on me dounait le salicylate de soude à la dose de 6.0 par jour que je n'ai pu supporter que deux jours. J'avais commencé à vomir tout ce que je prenais, même l'ean même la glace; et la fièvre continuait et la transpiration est devenue si abondante, que j'étais obligé de changer quatre fois, chaque nuit mon linge et mon lit. Maintenant des donleurs atroces rhumatoïdes ont apparu dans différentrs articulations avee un peu de gonflement. Le séro-diagnostic de $\nabla i d a l$ était négatif le 1le jour. Mon mal s'empirait de jour en jour, la fièvre continuiait, $j$ 'avais une toux sèche et je transpirai toujours beaucoup.

Le 18e jour on a cherché l'agglutination pour la fièvre de Malt, qui fut négative. Après avoir eu quelques frissons du 12 au 18 jour, la fièvre a commencé à baisser un peu par moment et dépuis elle a pris une forme ondnlante, sans jamais revenir à la marche normale et cette marche fébrile a duré peu à peu 11 mois. (Voici, Messieurs, la feuille de température de 180 jours.)

Les douleurs rhumatoides continuaient; c'était tantôt une articulation tantôt une autre; sur les surfaces osseuses, il se produisait des plaques sensibles et douloureuses avec un peu de gonflement comme la périosiste. Vers le 40e jour de ma maladie apparaissait une éruption qui a commencé d'abord par la face et quelques jours après, tout le corps était envahi par cette éruption. Vers la fin du $2 \mathrm{e}$ mois une épidymoorchite d'abord à gauche ensuite à droite fait son apparition. A ce moment on fait le séro-diagnostic, au point de vue de la fièvre de Malt. 
Cette fois-ci l'agglutination est positive et le diagnosicic de la fièvre de Malt est accepté. par les médecins. Tous les symptômes resistent d'une façon étrange à tout traitement. La fièvre, les sueurs, les douleurs, les vomissements continuent. Au commencement du $7 \mathrm{e}$ mois des complications occulaires apparaissent et depuis elles continuent. D'abord c'est une épisclérite suivie d'une iritis, qui évoluent en 2 à 3 mois. Après la guérison d'un oeil, quelques jours après, l'autre oeil se prend et ainsi de suite ça continue depuis un an."

Iei s'arrête la note, qui nous a été remise par Mr. le professeur et nous continuerons notre propre observation. L'aspect de l'éruption généralisée à la face et dans tout le corps présentait les caractères suivants: (voici les photographies de cette éruption) on voyait de petites élevures papuleuses de la grandeur d'une tête d'épingle jusqu'à celle d'une lentille, la plupart ayant à leur centre une miniscule vésiculo pustulette à peine saillante, d'autres papules ont à leur centre des petites vésicules nettement visibles et saillantes et d'autres éléments sont purement papuleux recouverts d'une petite squame. Ces petites élevures papuleuses guérissent spontanément en laissant des petites taches très pigmentées ou de toutes petites cicatrices centrales de la grandeur d'une tête d'épingle entourées d'une auréole pigmentée. On voit parmi ces élements la transformation de ces vésiculo-pustulettes centrales en croûtes, qui en s'éliminant laissent des parties pigmentées dépressives comme dans la variole et des petites cicatrices centrales; les uns laissant des pigmentations, persistantes, d'autres des pigmentations passagères. On voit aussi d'autres éléments beaucoup plus grands de la grandeur d'une lentille ayant la forme d'une pustule d'acné. Ces éléments sont isolés ou confluents en placards plus ou moins grands et on voit des pustules isolées ou confluentes saillantes vers la surface d'un demi à un millimètre et laissant après leur disparition des placards pigmentés très foncés. La face et tout le corps presque était couvert de ces éléments petits et grands mêlés ensemble et on voyait aussi sur les deux amygdales deux ulcérations. Cette éruption papuleuse par places noduleuses, par places vésiculo-pustuleuses, crô̂teuses par places ulcerées, de coloration violacée, d'apparence par places tubercoloỉde (par ses éléments nécrotiques) par places syphilitiques (par ses éléments papuleux) dure depuis 16 mois, sans interruption, des éléments disparaissent laissant des pigmentations; d'autres éléments nouveaux apparaissent. Les ulcères de la gorge ont disparu, d'autres lésions érythémateuses, légèrement bulleuses ou exulcérées ont apparu aux muqueuses buccales et labiales.

On reste très perplexe en voyant le malade. On se demande, estce la syphilis? les éléments papuleux pigmentés y font penser; mais le manque d'adénopathie; la fièvre continue depuis un an, la continuation depuis un an et quatre mois de cette éruption, la disparition et l'apparition incessante de ces éléments depuis plus d'un an, l'aspect cicatriciel necrotique de plusieurs de ces élements font penser à la tuberculose; c'est-à- 
dire aux tuberculides; mais la conservation d'une bonne santé relative après tant de mois de fièvre, tant d'éruptions, et tant de seuffrances font aussi éloigner le diagnostic de tuberculose. Comme vous le verrez tout de suite toutes ces éruptions de la face et du corps ont disparu en laissant des pigmentations et des circatrices, il y a à présent peu d'éléments nouveaux. Finalement nous avons pensé à la sporotrichose et nous avons eu recours aux cultures pour avoir un diagnostic décisif. Nous avons piqué un nodule vésiculo-pustuleux de la jambe, et avec le bout d'une pipette pasteur sterilisée, nous avons pris un pen de sécretion et nous avons placé dans un tube stérilisé, dans lequel nous avons mis un peu du liquide du milieu nutritif de $\mathrm{Sab}$ ou raud; c'est un véritable triomphe de la bactériologie, dans 4 jours nous avons obtenu d'emblée des cultures pures de sporotrichose, que nous vous placerons sous le microscope, et un diagnostic sûr ponvait être mis chez notre cher malade, qui a souffert pendant un an et demi; il s'agissait de septicémie sporotrichosique, il s'agissait de sporotrichose. Nous avons obtenu les cultures pures de sporotrichose facilement par une simple piqure d'unc de ces lésions; elles sont typiques, caractéristiques, la périphérie blanche rayonnée, le centre foncé et noirci; à travers le tube vous verrez sous le microscope les mycéliums filamenteux et les spores sporotrichosiques.

L'oculiste, qui a soigné notre cher Confrère, sans connaître le diagnostic de sporotrichose, a administré le iodure, et comme notre Confrère a une intolérance contre le iodure c'est la iodalise qu'il prenait de temps en temps et chaque fois qu'il prenait un peu de iodure notre malade dit qu'il avait des améliorations surtout du côté de l'oeil; mais après deux à trois semaines d'emploi, il cessait par suite de son intolérance contre le iodure, pour le reprendre après quelque temps, d'une façon très irrégulière et une quantité insuffissante.

L'examen microscopique du petit nodule que nous arons excisé de notre cher malade et que nous avons placé sous le microscope démontre les altérations tel que les auteurs français. les ont décrites pour la Sporotrichose. A l'épiderme il y a hypertrophie de la couche cornée et granuleuse et hypertrophie et hyperplasie de la couche épineuse avec un leger œedeme inter et intracellulaire plus prononcé au centre de la tumeur. Au derme on voit une tumeur cellulaire s'étendant depuis la base de l'épiderme jusqu'à l'hypoderme. A la péripherie de cette tumeur, aspect syphiloïde, hypertrophie et hyperplasie des cellules périthéliales et épaississement de parais des vaisseaux, hypertrophie et hyperplasie des cellules conjonctives du tissu intervasculaire, en s'approchant de la partie centrale il y a outre les altérations mentionnées aspect tuberculoïde, infiltrations périvasculaires et intervascu- 
laires d'amas de cellules plasmatiques, mêlées avec des cellules épithéloïdes et ça et là des cellules géantes disséminées mais on ne voit pas comme dans la tuberculose des follicules tuberculeux des cellules géantes par groupes de 4 à 5 et tout à fait au centre de la tumeur il y a la partie supérieure du derme grande leucocytose polynucléaires mêlés avec les autres éléments cellulaires mentionnés et à la partie inférieure du derme et à l'hypoderme une véritable masse de leucocytes polynucléaires un abcès microscopique ou petite gomme sous-cutanée en roie de formation tel qu'il a été décrit dans la sporotrichose; naturellement ce petit abcès microscopique en s'ouvrant plus tard vers la surface, se transforme en petit croûte centrale profonde, qui en s'éliminant laisse une cicatrice pigmentée persistante, tel que vous le voyez dans les multiples éléments acneïformes. En terminant nous remercions encore Monsieur le Professeur et à présant que grâce aux cultures pures de sporotrichose, le diagnostic est sûrement posé; nous esperons que notre cher malade fera tout son possible pour supporter à hante dose: le iodure nous lui avons prescrit le iodure Souffron, qui semble être le mieux toléré s'il n'est pas supporté nous aurons recours aux injections de iodipine et nous lui souhaitons une prompte guérison par le iodure. Nous aurons l'honneur de vous communiquer les résultats définitifs ou plutôt Mr. le professeur nous fera le plaisir de le faire lui-même. Nous derons ajouter qu'après avoir terminé cette étude nous avons pris ultérieurement du sang de notre cher malade et nous arons fait les examens suivants: 1. la séro-agglutination au point de vue de la fièvre de Malt faite avec le sérum du malade inactivé en chauffant $56^{\circ}$ pendant $1 / 2$. heure avec une dilution de culture de micrococcus, Méli tensis à $1 / 50$ a donné un résultat négatif, 2. un séro-diagnostic au point de vue de la syphilis faite par la méthode de Porges a donné un résultat négatif, 3. une séro-agglutination au point de vue de la sporotrichose d'après la méthode de $\mathrm{Vidal}$ et $\mathrm{Abrami}$ avec une solution diluée de spores d'une vielle culture de sporotrichose, d'anciens cas de sporotrichoses que nous avions précédemment publiées et qui a donné $1 / 300$ un résultat fortement pos itif. De façon que de notre malade actuel, nous arons obtenu de 
sa lésion nodulopustuleuse de la jambe une culture pure de sporotrichose et du sérum de son sang une séro-agglutination fortement positive à $1 / 300$, qui ne peut laisser dans ce cas aucun doute sur le diagnostic de sporotrichose. 